This item was submitted to Loughborough's Research Repository by the author.

Items in Figshare are protected by copyright, with all rights reserved, unless otherwise indicated.

\title{
Power-efficient resource allocation in NOMA virtualized wireless networks
}

PLEASE CITE THE PUBLISHED VERSION

http://dx.doi.org/10.1109/GLOCOM.2016.7842162

PUBLISHER

(c) IEEE

VERSION

AM (Accepted Manuscript)

LICENCE

CC BY-NC-ND 4.0

REPOSITORY RECORD

Dawadi, Rajesh, Saeedeh Parsaeefard, Mahsa Derakhshani, and Tho Le-Ngoc. 2019. "Power-efficient Resource Allocation in NOMA Virtualized Wireless Networks". figshare. https://hdl.handle.net/2134/22338. 


\title{
Power-Efficient Resource Allocation in NOMA Virtualized Wireless Networks
}

\author{
Rajesh Dawadi*, Saeedeh Parsaeefard ${ }^{\dagger}$, Mahsa Derakhshani $^{\ddagger}$, Tho Le-Ngoc* \\ * Department of Electrical \& Computer Engineering, McGill University, Montreal, QC, Canada \\ $\dagger$ Iran Telecommunication Research Center, Tehran, Iran \\ $\ddagger$ Wolfson School of Mechanical, Electrical \& Manufacturing Engineering, Loughborough University, UK \\ Email: rajesh.dawadi@mail.mcgill.ca; s.parsaeifard@itrc.ac.ir; m.derakhshani@lboro.ac.uk; tho.le-ngoc@mcgill.ca
}

\begin{abstract}
In this paper, we address a power-efficient resource allocation problem in virtualized wireless networks (VWNs) using non-orthogonal multiple access (NOMA). In this set-up, the resources of one base station (BS) are shared among different service providers (slices), where the minimum reserved rate is considered for each slice for guaranteeing their isolation. The formulated resource allocation problem aiming to minimize the total transmit power subject to the isolation constraints is non-convex and suffers from high computational complexity. By applying complementary geometric programming (CGP) to convert the non-convex problem into the convex form, we develop an efficient iterative approach with low computational complexity to solve the proposed problem. Illustrative simulation results on the performance evaluation of VWN using OFDMA and NOMA indicate significant performance improvement in the VWN when NOMA is used.
\end{abstract}

Index Terms-Complementary geometric programming, NOMA, 5G, next generation wireless network, resource allocation, virtualized wireless networks.

\section{INTRODUCTION}

The current trend of the increasing demand for higher data rates has led to the crunch of the available spectrum in wireless networks. Moreover, the wireless service providers face a challenge in reducing the operational costs of the wireless infrastructure. As such, various techniques such as massive MIMO, virtualization, non-orthogonal multiple access have been envisioned for the next generation of wireless network in order to address these challenge [1]. Virtualization of wireless networks is a promising technique, in which the physical wireless infrastructure is shared among multiple service providers (SPs), also called slices [2]. Aiming to increase the spectrum and infrastructure efficiency, the main issue in a virtualized wireless network (VWN) is to prevent the harmful effects of users of one slice to the users of other slices, which is captured by the concept of isolation among slices. To guarantee the quality of service (QoS) requirements of users of each slice, different forms of static and dynamic resource allocation have been proposed [3] - [4], calling for an efficient resource allocation algorithm.

For instance, in [5], interactions among slices, network operator, and users are studied by an auction. A novel admission control policy is proposed in [6] by considering the channel state information (CSI) of users in each slice to support the QoS of users. [7] proposes an opportunistic algorithm to allocate the resources to virtual operators. [8] investigates the advantages of full-duplex transmission relay in VWN. [9] studies the effects of deploying a large number of antennas in VWN to improve the total performance. However, more spectrum efficient approaches are required in a VWN due to the challenges in providing the isolation among slices.

Non-orthogonal multiple access (NOMA) has been recently introduced as an effective approach to increase spectrum efficiency and provide massive connectivity [10], [11]. Compared to the existing multiple access techniques such as OFDMA, via NOMA, users share the entire spectrum and are rather allocated different power allocation coefficients. Since the users share the time and frequency resources, sophisticated techniques for decoding the superimposed signal need to be implemented at the receiver. By implementing successive interference cancellation (SIC), the receiver iteratively subtracts the strongest signal from the superimposed signal and decodes the intended signal [12]. In contrast, in OFDMA, the users are allocated different sub-carriers, which effectively removes interference among users by exclusive sub-carrier allocation within a cell. The important question in this scenario is whether NOMA can improve the spectrum efficiency compared to OFDMA. There has been a significant research interest in this context. For instance, [13] compares the system level performance of the NOMA scheme with different mechanisms for power allocation including the user grouping based on their channel gains and equal power allocation to all users. The authors propose a sub-optimal power allocation scheme called fractional transmit power allocation (FTPA) that is similar to the transmission power control mechanism in LTE. Similarly, [14] analyzes the performance of NOMA compared to OFDMA for the cellular up-link setup. The optimization problem of this work includes the minimum required throughput of each user as a constraint. It has been shown that the performance of the system in the cell-edge is significantly improved in the case of NOMA compared to OFDMA. Similarly, [15] proposes an enhanced proportional fairness scheme based on NOMA and shows the improvement of cell throughput by up to $28 \%$ compared to OFDMA scheme. In [16], a power allocation problem for the downlink transmission of NOMA system is formulated 
and solved by applying difference of convex functions (DC) programming. In order to develop the proposed algorithm, the greedy user selection approach is used to assign users to subcarriers, and then, DC approximation is applied to allocate power for each user.

In this paper, we investigate the use of NOMA in the VWN to improve the network performance in terms of power efficiency. The objective is to minimize the total transmit power in a VWN, while maintaining the minimum required capacity for each slice. Since the original problem is non-convex and computationally intractable, we use the approach of complementary Geometric programming (CGPA) and arithmetic-geometric mean inequality (AGMA) to convert it into an efficient algorithm [17], [18]. The simulation results demonstrate that NOMA is more power-efficient than OFDMA in various scenarios. Specifically, the power efficiency is improved by up to $45-54 \%$ with NOMA as compared to OFDMA.

The rest of this paper is organized as follows. In Section II, the system model and problem formulation are discussed. Section III explains the proposed algorithm for both NOMA and OFDMA. Section IV presents the simulation results followed by the conclusion in Section V.

\section{System Model}

Consider the downlink transmission of a VWN with a single base station (BS) that serves a set of slices (i.e., $\mathcal{S}$ ), in which each slice $s \in \mathcal{S}$ has its own set of users denoted by $\mathcal{K}_{s}$. The total number of users in the system is given by $K=\sum_{s \in \mathcal{S}} K_{s}$. To provide the isolation among slices, the VWN should preserve a minimum required rate per each slice $s$, denoted by $R_{s}^{\text {rsv }}$. We consider the following two transmission modes for the VWN:

- Non-orthogonal multiple access (NOMA) where the whole frequency band of interest is shared among users,

- Orthogonal frequency division multiple access (OFDMA) where the specific bandwidth is divided into a set of sub-carriers denoted by $\mathcal{N}$ and each sub-carrier can be allocated to a maximum of one user at a time.

In this paper, our focus is to compare the power efficiency of these two approaches for our system model. We assume that the bandwidth $B$ is divided into a set of sub-carriers $\mathcal{N}=$ $\{1, \cdots N\}$, and the channel gain from the BS to the user $k_{s}$ in slice $s$ and in sub-carrier $n$ is

$$
h_{k_{s}, n}=\chi_{k_{s}, n} d_{k_{s}}^{-\lambda},
$$

where $\chi_{k_{s}, n}$ is the fading coefficient, $d_{k_{s}}>0$ is the distance of the user $k_{s} \in \mathcal{K}_{s}$ to the BS normalized to the cell radius and $\lambda$ is the path loss exponent.

\section{A. NOMA}

When the BS applies NOMA for downlink transmission to users, first, all users are indexed based on their channel gains in an increasing order such as $\left|h_{1, n}\right|<\left|h_{2, n}\right|<$ $\cdots<\left|h_{K, n}\right|, \forall n \in \mathcal{N}$. By developing successive interference cancellation (SIC), the user $k_{s}$, with index $i$, can successively remove the interference of all users $j \neq i$ if $j<i$, at subcarrier $n$. For the rest of the users, i.e., users with indices $j>i$, the interference cannot be removed. Consequently, the received SINR at the user $k_{s}$, with index $i$ at the sub-channel $n$, is given by

$$
\gamma_{i, n}^{\mathrm{NOMA}}=\frac{\beta_{i, n} h_{i, n}}{\sigma^{2}+h_{i, n} \sum_{s \in \mathcal{S}} \sum_{j=i+1}^{K} \beta_{j, n}},
$$

where $\beta_{i, n}$ is the power allocation coefficient for the user at the $i^{\text {th }}$ index and sub-carrier $n$. Moreover, $\sigma^{2}$ is the noise power, which is assumed to be equal for all users. The rate of user $k_{s}$, with index $i$, at the sub-carrier $n$ is

$$
\begin{aligned}
R_{k_{s}, n}^{\mathrm{NOMA}} & =R_{i, n}=\log _{2}\left(1+\gamma_{i, n}^{\mathrm{NOMA}}\right) \\
= & \log _{2}\left(1+\frac{\beta_{i, n} h_{i, n}}{\sigma^{2}+h_{i, n} \sum_{s \in \mathcal{S}} \sum_{j=i+1}^{K} \beta_{j, n}}\right)
\end{aligned}
$$

Each slice $s \in \mathcal{S}$ in the VWN has a minimum reserved rate of $R_{s}^{\text {rsv }}$ in order to support the QoS requirement of the users, which can be expressed as

$$
\mathrm{C} 1: \sum_{k_{s} \in \mathcal{K}_{S}} \sum_{n \in \mathcal{N}} R_{k_{s}, n}^{\mathrm{NOMA}} \geq R_{s}^{\mathrm{rsv}}, \quad \forall s \in \mathcal{S} .
$$

\section{B. OFDMA}

We consider an OFDMA system where the total available frequency is divided into $n \in \mathcal{N}$ sub-carriers and if $\alpha_{k_{s}, n}$ is the sub-carrier allocation indicator for the sub-carrier $n$ and user $k_{s}$ in slice $s \in \mathcal{S}$, then

$$
\alpha_{k_{s}, n}= \begin{cases}1, & \text { if sub-carrier } n \text { is allocated to user } k_{s}, \\ 0, & \text { otherwise. }\end{cases}
$$

Due to the OFDMA exclusive sub-carrier assignment, we have a constraint on $\alpha_{k_{s}, n}$ as

$$
\text { C2: } \sum_{\forall s} \sum_{\forall k_{s}} \alpha_{k_{s}, n} \leq 1, \quad \forall n \in \mathcal{N} .
$$

The received SINR at the user $k_{s}$ at sub-carrier $n \in \mathcal{N}$ and in slice $s \in \mathcal{S}$ is

$$
\gamma_{k_{s}, n}^{\text {OFDMA }}=\frac{P_{k_{s}, n} h_{k_{s}, n}}{\sigma^{2}},
$$

Hence, the rate of user $k_{s}$ at sub-carrier $n$ is

$$
R_{k_{s}, n}^{\text {OFDMA }}=\alpha_{k_{s}, n} \log _{2}\left(1+\gamma_{k_{s}, n}^{\text {OFDMA }}\right) .
$$

In this case, the minimum reserved rate of each slice is represented as

$$
\text { C3: } \sum_{k_{s} \in \mathcal{K}_{S}} \sum_{n \in \mathcal{N}} R_{k_{s}, n}^{\mathrm{OFDMA}} \geq R_{s}^{\mathrm{rvv}}, \quad \forall s \in \mathcal{S} .
$$

Consider $\boldsymbol{\beta}=\left[\boldsymbol{\beta}_{1}, \ldots, \boldsymbol{\beta}_{S}\right]$ as the vector of power allocation coefficients of all users in all slices in NOMA, where $\boldsymbol{\beta}_{s}=\left[\boldsymbol{\beta}_{k_{s}}\right]_{k_{s}=1}^{K_{s}}$ and $\boldsymbol{\beta}_{k_{s}}=\left[\beta_{k_{s}, 1}, \cdots, \beta_{k_{s}, N}\right]$, respectively. Similarly, for the OFDMA case, the power allocation vector of 
the system can be represented as $\mathbf{P}=\left[\mathbf{P}_{1}, \cdots, \mathbf{P}_{S}\right]$, where $\mathbf{P}_{s}=\left[\mathbf{P}_{k_{s}}\right]_{k_{s}=1}^{K_{s}}$ and $\mathbf{P}_{k_{s}}=\left[P_{k_{s}, 1}, \cdots, P_{k_{s}, N}\right]$. Also, the sub-carrier allocation vector of the system can be represented as $\boldsymbol{\alpha}=\left[\boldsymbol{\alpha}_{1}, \ldots, \boldsymbol{\alpha}_{S}\right]$, where $\boldsymbol{\alpha}_{s}=\left[\boldsymbol{\alpha}_{k_{s}}\right]_{k_{s}=1}^{K_{s}}$ and $\boldsymbol{\alpha}_{k_{s}}=$ $\left[\alpha_{k_{s}, 1}, \cdots, \alpha_{k_{s}, N}\right]$.

Now, for the case of NOMA, the optimization problem to minimize the total transmit power can be expressed as

$$
\min _{\boldsymbol{\beta}} \sum_{s \in \mathcal{S}} \sum_{k_{s} \in \mathcal{K}_{S}} \sum_{n \in \mathcal{N}} \beta_{k_{s}, n}
$$

subject to : $\mathrm{C} 1$.

For the case of OFDMA, the corresponding resource allocation problem is

$$
\min _{\mathbf{P}, \boldsymbol{\alpha}} \sum_{s \in \mathcal{S}} \sum_{k_{s} \in \mathcal{K}_{S}} \sum_{n \in \mathcal{N}} \alpha_{k_{s}, n} P_{k_{s}, n}
$$

subject to : $\mathrm{C} 2-\mathrm{C} 3$.

The proposed algorithm to solve the optimization problem is described in the subsequent section for both NOMA and OFDMA schemes.

\section{Proposed Algorithm}

The formulated optimization problems for both cases of NOMA and OFDMA in (6) and (7) are non-convex and solving them is challenging. To develop an efficient algorithm to solve (6), we deploy an iterative framework of successive convex approximation, in which the non-convex function is transformed into a convex one in each iteration. For this transformation, we apply the complementary geometric programming (CGP) and variable relaxation to convert (6) into the geometric programming (GP) formulation. We refer the interested readers for the background of CGP to [19]-[21]. For (7), we apply the relaxation technique to convert binary variables into continuous ones. Then we use Lagrange dual function which has been widely utilized for solving OFDMA-based resource allocation problems [6], [22].

\section{A. Iterative Algorithm for NOMA-based Resource Allocation}

$$
\begin{aligned}
& \text { Considering } R_{k_{s}, n}^{\mathrm{NOMA}}=\log _{2}\left(1+\gamma_{i, n}^{\mathrm{NOMA}}\right) \text { as } \\
& \widetilde{R}_{k_{s}, n}^{\mathrm{NOMA}}= \\
& \quad \log _{2}\left(\frac{\sigma^{2}+h_{i, n} \sum_{s \in \mathcal{S}} \sum_{j=i+1}^{K} \beta_{j, n}+\beta_{i, n} h_{i, n}}{\sigma^{2}+h_{i, n} \sum_{s \in \mathcal{S}} \sum_{j=i+1}^{K} \beta_{j, n}}\right) .
\end{aligned}
$$

From the above, $\mathrm{C} 1$ can be rewritten as

$$
\begin{aligned}
& \prod_{i \in \mathcal{K}_{s}} \prod_{n \in \mathcal{N}}\left(\frac{\sigma^{2}+h_{i, n} \sum_{s \in \mathcal{S}} \sum_{j=i+1}^{K} \beta_{j, n}}{\sigma^{2}+h_{i, n} \sum_{s \in \mathcal{S}} \sum_{j=i+1}^{K} \beta_{j, n}+\beta_{i, n} h_{i, n}}\right) \\
& \quad \leq 2^{\left(-R_{s}^{\mathrm{rsv}}\right)}, \quad \forall s \in \mathcal{S} .
\end{aligned}
$$

To apply the CGP, consider $t_{1}$ as the iteration number. In each iteration $t_{1}$, the non-convex function should be approximated to its convex counterpart. Based on the structure of $\widetilde{R}_{k_{s}, n}^{\mathrm{NOMA}}$, we can apply AGMA approximation to propose the monomial approximation of $\widetilde{R}_{k_{s}, n}^{\mathrm{NOMA}}$. At iteration $t_{1}, \widetilde{R}_{k_{s}, n}^{\mathrm{NOMA}}$ can be $\overline{\text { Algorithm } 1 \text { : Iterative Algorithm Based on CGP for NOMA }}$

Initialization: Set $t_{1}=1, \boldsymbol{\beta}\left(t_{1}\right)=[\mathbf{1}]$, where $\mathbf{1}$ is a vector $C^{1 \times K}$.

\section{Repeat:}

Step 1: Update $s_{i, n}\left(t_{1}\right), g_{j, n}\left(t_{1}\right), r_{i, n}\left(t_{1}\right)$, and $z_{i, n}\left(t_{1}\right)$ from (11)-(14),

Step 2: Find optimal $\boldsymbol{\beta}^{*}\left(t_{1}\right)$ from (15) via CVX [23],

Until: $\left\|\boldsymbol{\beta}^{*}\left(t_{1}\right)-\boldsymbol{\beta}^{*}\left(t_{1}-1\right)\right\| \leq \varepsilon_{1}$.

approximated as $\widetilde{R}_{k_{s}, n}^{\mathrm{NOMA}}=\log _{2}\left(x_{i, n}^{-1}\left(t_{1}\right)\right)$ where $x_{i, n}\left(t_{1}\right)$, for all $i$, is given by,

$$
\begin{gathered}
x_{i, n}\left(t_{1}\right)=\left(\sigma^{2}+h_{i, n} \sum_{s \in \mathcal{S}} \sum_{j=i+1}^{K} \beta_{j, n}\right)\left(\frac{\sigma^{2}}{s_{i, n}\left(t_{1}\right)}\right)^{-s_{i, n}\left(t_{1}\right)} \times \\
\prod_{\forall s, j=i+1}^{K}\left(\frac{h_{i, n} \beta_{j, n}\left(t_{1}\right)}{g_{j, n}\left(t_{1}\right)}\right)^{-g_{j, n}\left(t_{1}\right)}\left(\frac{\beta_{i, n}\left(t_{1}\right) h_{i, n}}{r_{i, n}\left(t_{1}\right)}\right)^{-r_{i, n}\left(t_{1}\right)},
\end{gathered}
$$

where for all $i$ and $n \in \mathcal{N}$,

$$
\begin{aligned}
s_{i, n}\left(t_{1}\right) & =\frac{\sigma^{2}}{z_{i, n}\left(t_{1}\right)}, \\
g_{j, n}\left(t_{1}\right) & =\frac{\beta_{j, n}\left(t_{1}-1\right) h_{j, n}}{z_{i, n}\left(t_{1}\right)}, \\
r_{i, n}\left(t_{1}\right) & =\frac{\beta_{i, n}\left(t_{1}-1\right) h_{i, n}}{z_{i, n}\left(t_{1}\right)}, \\
z_{i, n}\left(t_{1}\right) & = \\
\sigma^{2}+h_{i, n} & \sum_{s \in \mathcal{S}} \sum_{j=i+1}^{K} \beta_{j, n}\left(t_{1}-1\right)+h_{i, n} \beta_{i, n}\left(t_{1}-1\right) .
\end{aligned}
$$

Considering (10)-(14), the optimization problem (6) at iteration $t_{1}$ is approximated to the following convex optimization problem

$$
\begin{aligned}
& \min _{\boldsymbol{\beta}\left(t_{1}\right)} \sum_{i=1}^{K} \sum_{n=1}^{N} \beta_{i, n}\left(t_{1}\right) \\
& \text { subject to: }(10)-(14) \\
& \prod_{i \in \mathcal{S}} \prod_{n \in \mathcal{N}} x_{i, n}\left(t_{1}\right) \leq 2^{\left(-R_{s}^{\text {rsv }}\right)}, \quad \forall s \in \mathcal{S} .
\end{aligned}
$$

The overall iterative algorithm to solve (6) based on the convex function (15) is presented in Algorithm 1.

\section{B. Dual Approach for OFDMA-based Resource Allocation}

Since (7) involves binary variables $\boldsymbol{\alpha}$, we first relax $\alpha_{k_{s}, n} \in$ $[0,1], \forall k_{s} \in \mathcal{K}_{s}, \forall s \in \mathcal{S}, \forall n \in \mathcal{N}$. Now, by considering $y_{k_{s}, n}=\alpha_{k_{s}, n} P_{k_{s}, n}$, the total rate of OFDMA can be rewritten as [6], [22],

$$
\widetilde{R}_{k_{s}, n}^{\text {OFDMA }}(\boldsymbol{\alpha}, \mathbf{y})=\alpha_{k_{s}, n} \log _{2}\left(1+\frac{y_{k_{s}, n} h_{k_{s}, n}}{\alpha_{k_{s}, n} \sigma^{2}}\right) .
$$

Note that the above expression belongs to a class of convex functions with the format of $f(a, b)=a \log (1+b / a)$ [24]. 


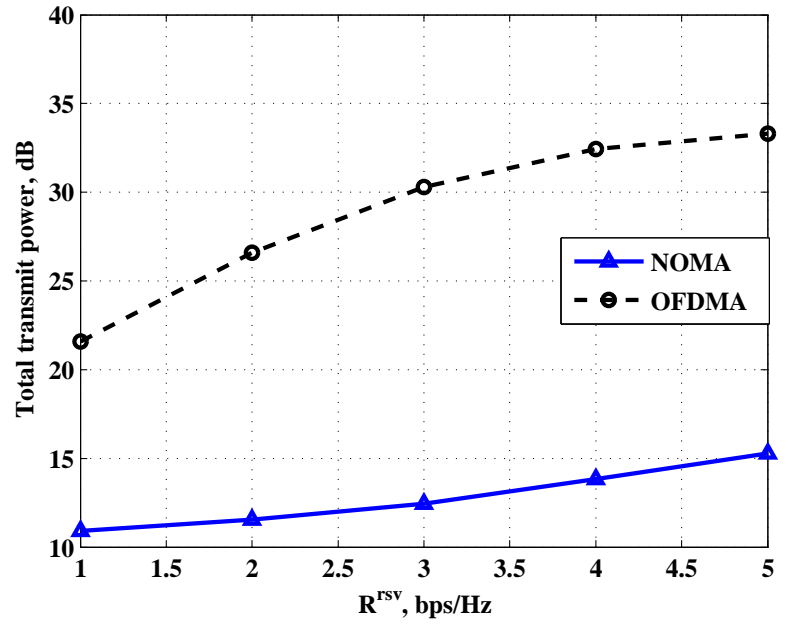

Fig. 1. Total transmit power versus $R^{\text {rsv }}$

Therefore, $\mathrm{C} 3$ can be written as

$$
\widetilde{\mathrm{C}} 3: \sum_{k_{s} \in \mathcal{K}_{S}} \sum_{n \in \mathcal{N}} \widetilde{R}_{k_{s}, n}^{\mathrm{OFDMA}}(\boldsymbol{\alpha}, \mathbf{y}) \geq R_{s}^{\mathrm{rvv}}, \quad \forall s \in \mathcal{S} .
$$

Consequently, (7) can be written as

$$
\begin{aligned}
& \min _{\mathbf{y}, \boldsymbol{\alpha}} \sum_{s \in \mathcal{S}} \sum_{k_{s} \in \mathcal{K}_{S}} \sum_{n \in \mathcal{N}} y_{k_{s}, n}, \\
& \text { subject to: } \mathrm{C} 2, \widetilde{\mathrm{C}} 3 .
\end{aligned}
$$

Proposition 1: Problem (18) is convex and can be solved using the Lagrange dual method. [6]

\section{Proof. See Appendix A.}

To solve the convex problem (18), the iterative algorithm based on the dual function can be applied with a low computational complexity as demonstrated in [6], [22] which is summarized in Algorithm 2.

\section{Simulation Results}

To study the performance of the proposed algorithm for NOMA and compare it with the OFDMA scheme, we simulate a scenario of a VWN with a single BS serving two slices each with $K_{s}=8$ users, where $K=\sum_{s \in \mathcal{S}} K_{s}$ and $R^{\text {rsv }}=R_{s}^{\text {rsv }}$ for all $s \in \mathcal{S}$. The users are randomly located (from a uniform distribution) within the whole area of interest unless otherwise stated. The total number of sub-carriers is taken to be $N=64$. The channel gains are derived according to the Rayleigh fading model. More specifically, $h_{k_{s}, n}=\chi_{k_{s}, n} d_{k_{s}}^{-\lambda}$ where $\lambda=3$ is the path loss exponent, $d_{k_{s}}>0$ is the distance between the BS and user $k_{s}$ normalized to the cell radius, and $\chi_{k_{s}}$ is the exponential random variable with mean of 1 . The results are taken over the average of 100 different channel realizations.

In Fig. 1, the total transmit power versus $R^{\text {rsv }}$ is depicted for both NOMA and OFDMA schemes. From Fig. 1, it is clear that the total transmit power increases with increasing $R^{\text {rsv }}$ for

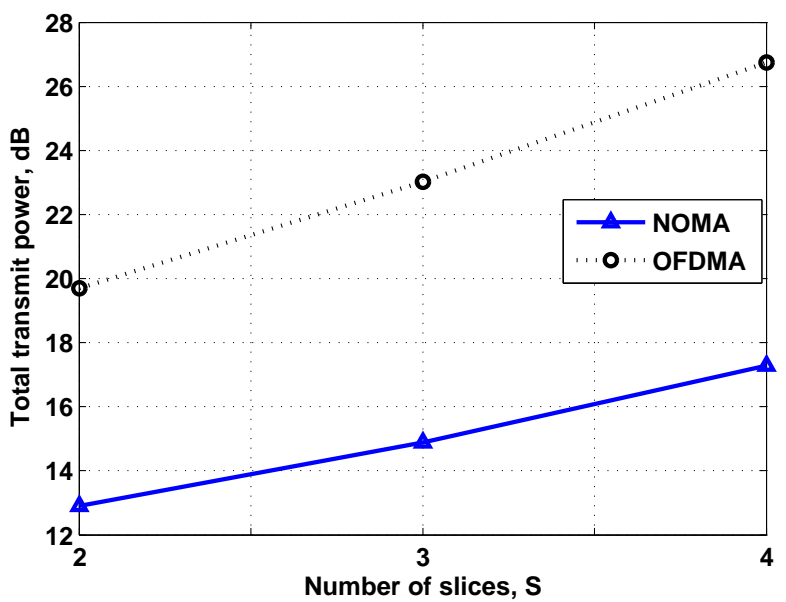

Fig. 2. Total transmit power versus $S$

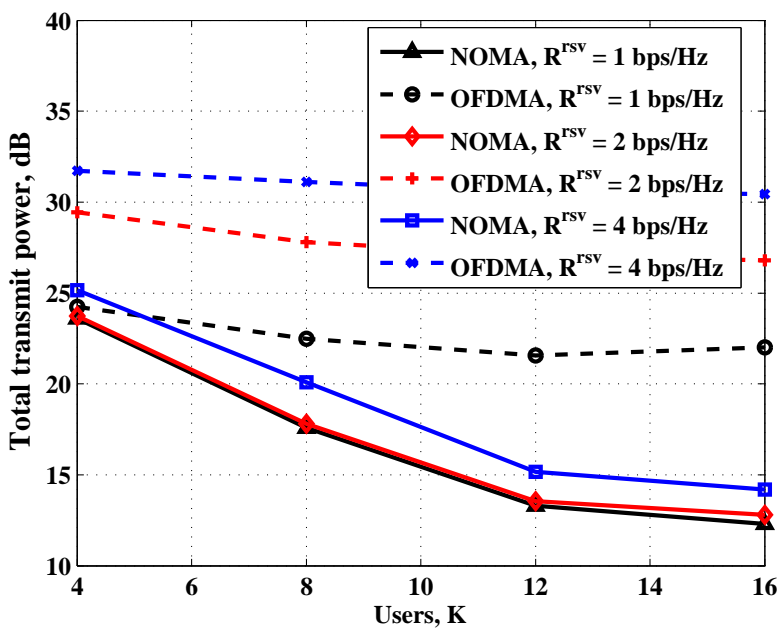

Fig. 3. Total transmit power versus $K$

both cases. It is because the BS needs to transmit at a higher transmit power to satisfy the minimum reserved rate per slice. However, the total transmit power in the case of OFDMA is higher than that in the case of NOMA, indicating that NOMA is more power efficient that OFDMA. Specifically, the total transmit power has been decreased by almost $45 \%$ from 22 $\mathrm{dB}$ to almost $12 \mathrm{~dB}$ at $R^{\mathrm{rsv}}=1 \mathrm{bps} / \mathrm{Hz}$ and by $54 \%$ from 33 $\mathrm{dB}$ to $15 \mathrm{~dB}$ at $R^{\mathrm{rsv}}=5 \mathrm{bps} / \mathrm{Hz}$, respectively, with NOMA as compared to OFDMA.

In Fig. 2, the total transmit power versus $S$ is plotted for both NOMA and OFDMA for $K=12$ and $R_{s}^{\mathrm{rsv}}=1 \mathrm{bps} / \mathrm{Hz}, \forall s \in \mathcal{S}$. As expected, the total transmit power increases with increasing the number of slices due to the rate reservation constraint per each slice. However, the total transmit power for OFDMA is significantly higher than the NOMA which demonstrates the power efficiency achieved via NOMA. 


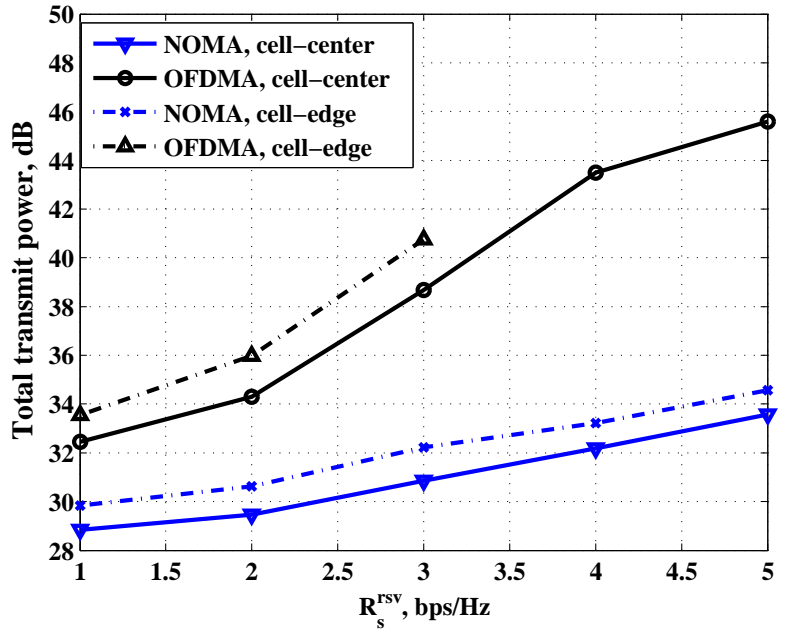

Fig. 4. Total transmit power versus $R^{\text {rsv }}$ for cell-edge and cell-center users

Fig. 3 plots the total transmit power versus $K$ for different $R^{\text {rsv }}$. From Fig. 3 and as expected from the multi-user diversity gain [25], it can be observed that the total transmit power decreases with increasing $K$ for a fixed $R^{\text {rsv }}$. Also, similar to Fig. 1, it is clear that the total transmit power is an increasing function of $R^{\text {rsv }}$, while it is higher in the case of OFDMA as compared to NOMA.

In Fig. 4, we study the effect of NOMA with non-uniform user distribution over the VWN for two scenarios, where in the first scenario, users are located close to the cell-center, i.e., the normalized distance, $d_{k_{s}} \in[0.1,0.7]$ and in the second scenario, the users are located close to the cell boundary, i.e. $d_{k_{s}} \in[0.8,1]$. Fig. 4 shows the total transmit power versus $R_{s}^{\mathrm{rsv}}$ for both OFDMA and NOMA. Based on the results in Fig. 4, the total transmit power with OFDMA is more than in the case of NOMA for both scenarios. Also, with increasing $R_{s}^{\text {rsv }}$, the total transmit power sharply increases for OFDMA as compared to that for NOMA, e.g., for $R_{s}^{\text {rvv }}>3 \mathrm{bps} / \mathrm{Hz}$. More importantly, via OFDMA, for the cell-edge scenario, there is no solution for the resource allocation problem for $R_{s}^{\text {rsv }}>3 \mathrm{bps} / \mathrm{Hz}$. However NOMA can reach the feasible solution by increasing the transmit power. This indicates the effectiveness of NOMA in achieving higher energy efficiency while preserving the isolation based constraints of the slices in the VWN.

\section{CONCLUSION}

In this paper, we investigated the power efficiency performance of NOMA compared to OFDMA for a virtualized wireless network (VWN). In particular, we formulated an optimization problem with the objective to minimize the transmit power, while supporting the minimum reserved rate per each slice to ensure effective isolation among users in the slices. Since the resource allocation problem is non-convex and suffers from high computational complexity, we developed the CGP and
AGMA approximation to propose the computationally tractable iterative algorithm. Via simulation results, we investigated the performance of the algorithm and compared it with the OFDMA scheme. Simulation results reveal that the proposed algorithm outperforms the OFDMA in terms of the required transmit power, specifically when most of users are located near the cell-edge and there is a diverse variation in the channel conditions. In a practical VWN deployment, the coverage of an area is provided by multiple BSs. Consequently, investigating the power efficiency of NOMA in multi-cell scenario is of interest, which remains as a future work of this paper.

\section{APPENDIX}

\section{A. Proof of Proposition 1}

In (16), $\widetilde{R}_{k_{s}, n}(\boldsymbol{\alpha}, \mathbf{y})$ is of the form $f(a, b)=a \log (1+b / a)$ which is a convex function and can be solved by the Lagrangian method [24].

The corresponding Lagrange function for (18) is

$$
\begin{aligned}
\mathcal{L}\left(\phi_{s}, \nu_{n}, \mathbf{y}, \boldsymbol{\alpha}\right)= & \sum_{\forall s, \forall k_{s}, \forall n} y_{k_{s}, n}+\sum_{\forall s} \phi_{s}\left(R_{s}^{\mathrm{rsv}}-\right. \\
& \left.\sum_{\forall k_{s}, n} \widetilde{R}_{k_{s}, n}^{\mathrm{OFDMA}}\right)+\sum_{\forall n} \nu_{n}\left(\sum_{s} \sum_{k_{s}} \alpha_{k_{s}, n}-1\right),
\end{aligned}
$$

where $\phi_{s}, \forall s \in \mathcal{S}$ and $\nu_{n}, \forall n \in \mathcal{N}$ are the Lagrange variables associated to $\widetilde{\mathrm{C}} 1$ and $\mathrm{C} 2$, respectively. Considering $\phi$ and $\nu$ as the vectors of the Lagrange variables for $\phi_{s}$ and $\nu_{n}, \forall s, \forall n$, respectively, the dual function for (19) is, [24]

$$
\mathcal{D}(\phi, \nu)=\min _{\mathbf{y}, \boldsymbol{\alpha}} \mathcal{L}(\phi, \nu, \mathbf{y}, \boldsymbol{\alpha}) .
$$

Thus, the dual problem can be written as

$$
\begin{aligned}
& \max _{\phi, \nu} \mathcal{D}(\phi, \nu) \\
& \text { subject to: } \phi>0 \& \boldsymbol{\nu}>0 .
\end{aligned}
$$

Since problem (18) is convex, the duality gap is zero and hence, the solution of the dual problem is equal to the solution of the primal problem [24]. Hence, by applying KKT conditions, the optimal power allocation for user $k_{s}$ in slice $s$ and sub-carrier $n$, i.e., $P_{k_{s}, n}^{*}$, is

$$
P_{k_{s}, n}^{*}=\left[\frac{\phi_{s}}{\ln (2)}-\frac{\sigma^{2}}{h_{k_{s}, n}}\right]_{0}^{P_{\max }},
$$

where, $[x]_{a}^{b}=\max \{\min \{x, b\}, a\}$. Also, the optimal sub-carrier allocation, $\alpha_{k_{s}, n}^{*}$, is

$$
\alpha_{k_{s}, n}^{*}= \begin{cases}0, & \frac{\partial\left(\mathcal{L}\left(\phi_{s}, \nu_{n}, \mathbf{y}, \boldsymbol{\alpha}\right)\right)}{\alpha_{k_{s}, n}^{*}}<0 \\ \in[0,1], & \frac{\partial\left(\mathcal{L}\left(\phi_{s}, \nu_{n}, \mathbf{y}, \boldsymbol{\alpha}\right)\right)}{\alpha_{k_{s}, n}^{*}, n}=0 \\ 1, & \frac{\left.\partial \mathcal{L}\left(\phi_{s}, \nu_{n}, \boldsymbol{\alpha}, \boldsymbol{\alpha}\right)\right)}{\alpha_{k_{s}, n}^{*}}>0\end{cases}
$$




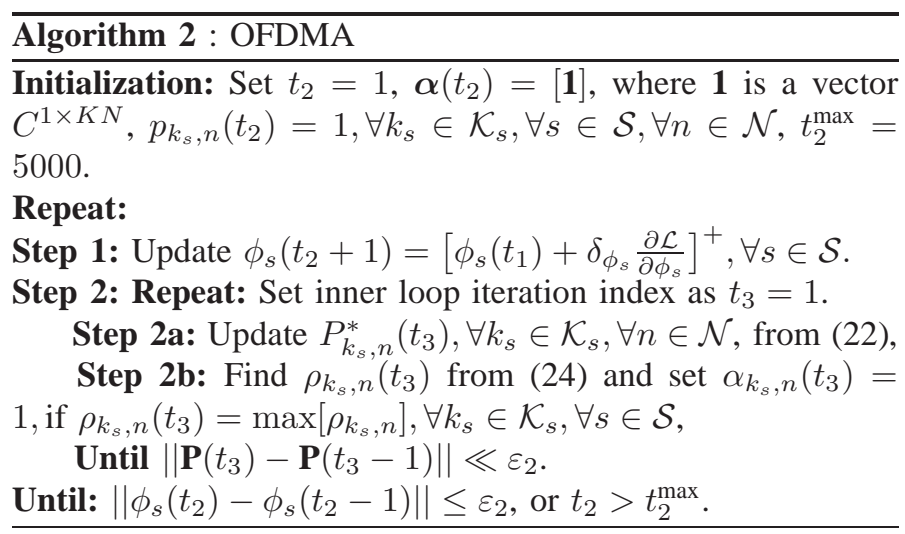

where,

$$
\begin{aligned}
& \frac{\partial\left(\mathcal{L}\left(\phi_{s}, \nu_{n}, \mathbf{y}, \boldsymbol{\alpha}\right)\right)}{\partial \alpha_{k_{s}, n}^{*}}= \\
& \nu_{n}-\phi_{s}\left(\log _{2}\left(1+\gamma_{k_{s}, n}\right)-\frac{\gamma_{k_{s}, n}}{\left(1+\gamma_{k_{s}, n}\right) \ln (2)}\right), \quad \forall s \in \mathcal{S}
\end{aligned}
$$

and $\gamma_{k_{s}, n}=\frac{y_{k_{s}, n} h_{k_{s}, n}}{\alpha_{k_{s}, n} \sigma}$. Now, from the KKT conditions, we have

$$
\rho_{k_{s}, n}=\phi_{s}\left[\log _{2}\left(1+\gamma_{k_{s}, n}\right)-\frac{\gamma_{k_{s}, n}}{\left(1+\gamma_{k_{s}, n}\right) \ln (2)}\right], \forall s \in \mathcal{S} .
$$

To satisfy the OFDMA exclusive sub-carrier allocation, $\alpha_{k_{s}, n}^{*}$ is chosen such that $\rho_{k_{s}, n}$ is maximum [26], mathematically represented as

$$
\alpha_{k_{s}, n}^{*}= \begin{cases}1, & k_{s}^{\prime}=\max _{\forall k_{s} \in \mathcal{K}_{s}, \forall s \in \mathcal{S}} \frac{\partial\left(\mathcal{L}\left(\phi_{s}, \nu_{n}, \mathbf{y}, \boldsymbol{\alpha}\right)\right)}{\partial \alpha_{k_{s}, n}^{*}} \\ 0, & k_{s} \neq k_{s}^{\prime} .\end{cases}
$$

The overall algorithm is described in Algorithm 2.

\section{REFERENCES}

[1] M. Agiwal, A. Roy, and N. Saxena, "Next generation 5G wireless networks: A comprehensive survey," IEEE Commun. Surveys Tuts., vol. 4, no. 99, pp. 1553-1563, Feb. 2016.

[2] R. Kokku, R. Mahindra, H. Zhang, and S. Rangarajan, "NVS: A substrate for virtualizing wireless resources in cellular networks," IEEE/ACM Trans. Netw., vol. 20, no. 5, pp. 1333-1346, Oct. 2012.

[3] K. Tan, H. Shen, J. Zhang, and Y. Zhang, "Enabling flexible spectrum access with spectrum virtualization," in IEEE Intl. Symp. on Dynamic Spectrum Access Networks (DYSPAN), Oct. 2012.

[4] C. Liang and F. Yu, "Wireless network virtualization: A survey, some research issues and challenges," IEEE Commun. Surveys Tuts., vol. 17, no. 1 , pp. $358-380,2014$.

[5] F. Fu and U. Kozat, "Stochastic game for wireless network virtualization," IEEE/ACM Trans. Netw., vol. 21, no. 1, pp. 84-97, Feb. 2013.

[6] S. Parsaeefard, V. Jumba, M. Derakhshani, and T. Le-Ngoc, "Joint Resource Provisioning and Admission Control in Wireless Virtualized Networks," in Proc. IEEE Wireless Commun. Netw. Conf. (WCNC), Mar. 2015, pp. $2020-2025$.

[7] M. Yang, Y. Li, D. Jin, J. Yuan, I. You, and L. Zeng, "Opportunistic sharing scheme for spectrum allocation in wireless virtualization," Soft Computing, vol. 18, no. 9, pp. 1685-1696, 2014. [Online]. Available: http://dx.doi.org/10.1007/s00500-014-1267-z

[8] G. Liu, F. Yu, H. Ji, and V. Leung, "Distributed resource allocation in full-duplex relaying networks with wireless virtualization," in Proc. IEEE Global Commun. Conf. (GLOBECOM), Dec. 2014, pp. 4959-4964.
[9] V. Jumba, S. Parsaeefard, M. Derakhshani, and T. Le-Ngoc, "Resource Provisioning in Wireless Virtualized Networks via massive-MIMO," IEEE Wireless Commun. Lett., vol. 4, no. 3, pp. 237-240, June 2015.

[10] Y. Saito, A. Kishiyama, T. Benjebbour, A. Nakamura, Li, and H. K., "Non-orthogonal multiple access (NOMA) for cellular future radio access," in Proc. IEEE Veh. Tech. Conf. (VTC), June 2013, pp. 1-5.

[11] NTT Docomo Inc., "5G Radio Access: Requirements, concept and technologies," July 2014.

[12] S. Sen, N. Santhapuri, R. R. Choudhury, and S. Nelakuditi, "Successive interference cancellation: A back-of-the-envelope perspective," in $A C M$ SIGCOMM Workshop on Hot Topics in Networks, 2010, pp. 171-176.

[13] A. Benjebbour, A. Li, Y. Saito, Y. Kishiyama, A. Harada, and T. Nakamura, "System-level performance of downlink NOMA for future LTE enhancements," in Proc. IEEE Global Commun. Conf. (GLOBECOM), Dec 2013, pp. 66-70.

[14] T. Takeda and K. Higuchi, "Enhanced user fairness using non-orthogonal access with SIC in cellular uplink," in Proc. IEEE Veh. Tech. Conf. (VTC), Sept 2011, pp. 1-5.

[15] X. Chen, A. Benjebbour, A. Li, and A. Harada, "Multi-user proportional fair scheduling for uplink non-orthogonal multiple access (NOMA)," in Proc. IEEE Veh. Tech. Conf. (VTC), May 2014, pp. 1-5.

[16] P. Parida and S. S. Das, "Power allocation in OFDM based NOMA systems: A DC programming approach," in Proc. IEEE Global Commun. Conf. (GLOBECOM), Dec 2014, pp. 1026-1031.

[17] S. Parsaeefard, R. Dawadi, M. Derakhshani, and T. Le-Ngoc, "Joint UserAssociation and Resource-Allocation in Virtualized Wireless Networks," IEEE Access, vol. 4, pp. 2738-2750, June 2016.

[18] M. Derakhshani, X. Wang, T. Le-Ngoc, and A. Leon-Garcia, "Airtime Usage Control in Virtualized Multi-Cell 802.11 Networks," in Proc. IEEE Global Commun. Conf. (GLOBECOM), Dec 2015, pp. 1-6.

[19] M. Avriel and A. C. Williams, "Complementary geometric programming," SIAM Journal on Applied Mathematics, vol. 19, no. 1, pp. 125-141, 1970.

[20] G. Xu, "Global optimization of signomial geometric programming problems," European Journal of Operational Research, vol. 233, no. 3, pp. $500-510,2014$.

[21] M. Chiang, "Geometric programming for communication systems," Commun. Inf. Theory, vol. 2, no. 1, pp. 1-154, July 2005.

[22] K. Seong, M. Mohseni, and J. M. Cioffi, "Optimal resource allocation for OFDMA downlink systems," in Proc. IEEE Intl. Symp. on Inf. Theory, July 2006, pp. 1394-1398.

[23] M. Grant and S. Boyd, "CVX: Matlab software for disciplined convex programming, version 2.1," http://cvxr.com/cvx, 2014.

[24] S. Boyd and L. Vandenberghe, Convex Optimization. Cambridge University Press, 2009.

[25] A. Goldsmith, Wireless Comunications. Cambridge University Press, 2004.

[26] N. Hassan and M. Assaad, "Resource allocation in multiuser OFDMA system: Feasibility and optimization study," in Proc. IEEE Wireless Commun. Netw. Conf. (WCNC), April 2009, pp. 1-6. 